\title{
Ischaemic stroke and thrombolysis: ask the onset time, not the age!
}

\author{
Silvia Cenciarelli • Tatiana Mazzoli • \\ Stefano Ricci
}

Received: 10 September 2012/ Accepted: 8 November 2012/Published online: 25 November 2012 (C) SIMI 2012

As of May 2012, indications for thrombolysis in acute ischaemic stroke were particularly limited [1]: patients had to be treated within $4.5 \mathrm{~h}$ from the onset, should be younger than 80 years old, should present with a moderate neurological deficit, and should not present important early ischaemic changes on admission head CT scan, and should not have any of the traditional contraindications for rt-PA therapy. As a consequence, it is not surprising that, despite the commendable efforts of many stroke clinicians, the number of treated patients was very small, with figures around 2-3\% of all strokes [2].

On 23 May 2012, during the European Stroke Conference in Lisbon, results of international stroke trial 3 (IST 3) were presented. The IST 3 is the largest randomised study on thrombolysis in acute ischaemic stroke ever done. The trial, whose complete results were subsequently published in Lancet on the 23 June 2012 [3], included 3,035 patients, without age or severity limits, randomised and treated within $6 \mathrm{~h}$ from symptoms onset. IST 3 is a non-profit trial, driven by researchers, with financial support from public agencies, randomized, with open treatment but blind assessment (PROBE design); it lasted 12 years (from 2000 to 2012). It is obvious that during this time many aspects changed in analysing and interpreting stroke trials results, and therefore some adjustments had been necessary during the trial conduct, to adequately study new information, particularly in the field of outcome evaluation. In fact, at the trial start a traditional cut off in Rankin scale (0-2 vs. 3-6) was chosen to evaluate the outcome, and from this cut

S. Cenciarelli · T. Mazzoli · S. Ricci $(\square)$

Unità Operativa di Neurologia, ASL 1 dell' Umbria, Centro

di Coordinamento IST 3 per l' Italia, Ospedali di Città di

Castello e Branca, Città di Castello, Italy

e-mail: stefano.ricci@asl1.umbria.it off a sample size of 3,100 patients was calculated, to show an absolute benefit of $4 \%$. In the following years, however, two facts prompted the steering committee to add further prognostic evaluations: the first one was ECASS 3 results publication [4], which were not conventionally significant for 2/3 Rankin cut-off, but were statistically significant when using a different cut off (0-1 vs. 2-6); these results caused a wide variation in clinical practice, moving the time limit of treatment from 3 to $4.5 \mathrm{~h}$. The second one was the re-evaluation of trials results by means of ordinal shift analysis [5], a statistical tool which uses all the information from the data, considering all the shifts from one to another grade in Rankin scale, and therefore is more powerful and sensitive for outcome evaluation in acute stroke. Therefore, the steering committee decided, much in advance of the database opening, to introduce these two new endpoints (Rankin cut-off $1 / 2$ and OSA), and to publish the whole statistical analysis plan [6], a procedure not very frequent in stroke trials; in this way, anyone could know the plans for data analysis and interpretation, with the researchers still blind to the actual results.

Further to the whole analysis of data, with the three aforementioned endpoints, a few hypotheses, based on clinical impressions and beliefs, had to be verified by IST 3 results: the earlier the treatment is given, the more effective it is (the concept of "time is brain"); rt-PA therapy is less effective and more dangerous in older and more severe patients.

From 2000 to 2012, 156 hospitals in 12 countries participated in IST 3; in Italy, 21 centres included 326 patients. $53 \%$ of patients were older than 80; on the whole, $95 \%$ did not fulfil the official licence criteria for rtPA use. At 6-month follow up, $37 \%$ of patients treated with thrombolysis and $35 \%$ of controls were alive and independent (Rankiin scale <3) (OR 1.13, CI 0.95/1.35, 
$p=0.18$ ), which means a not significant increase of 14 patients alive and independent out of 1,000 treated. OSA showed a clear benefit of $27 \%$ of thrombolysis ( $p=0.001) ; 29$ more patients out of 1,000 treated had a favourable outcome (Rankin $<2$, or ECASS 3 end-point), a result which is highly significant $(p=0.018)$. Early intracranial haemorrhages happened in $7 \%$ of cases and $1 \%$ of controls; this result is very similar to the figure obtained by the SITS- MOST register (7.3\%). 6-month mortality did not differ between the two groups. As opposed to common belief before IST 3, older and more severe patients had a higher benefit from thrombolysis, particularly when treated within $3 \mathrm{~h}$.

The updated Cochrane systematic review [7], which appeared in the same issue of Lancet, confirmed the results, reinforcing the concept that patients older than 80 years achieved similar benefit to those aged 80 years or younger, particularly when treated early.

In the editorial, which was published in the same Lancet issue [8], Leys and Cordonnier wrote "The key message of IST-3 and the updated meta-analysis is that many eligible patients from subgroups excluded by the European licence should now be given rt-PA. Every stroke patient should therefore be classed as a candidate for thrombolysis and managed as a medical emergency irrespective of age, severity, and clinical presentation....and the role of stroke and emergency physicians is now not to identify patients who will be given rt-PA, but to identify the few who will not."

No doubt there are still some points to clarify, as for instance the predictive factors for haemorrhage or better clinical response. Further analyses will be possible thanks to a single patient meta-analysis, which has been planned by all the Authors of trials on r-tPA in acute ischaemic stroke; preliminary results of this important review will be available during 2013 .
While waiting for this important information, we clinicians should do our best to treat as early as possible all patients with ischaemic stroke seeking our intervention. We have no longer to ask the age, but just the onset time!

Conflict of interest None.

\section{References}

1. SPREAD Italian National Guidelines for Stroke (2012) http:// www.spread.it accessed on 30-9-2012

2. Rudd AG, Hoffman A, Grant R, Campbell JT, Lowe D, On behalf of the intercollegiate working party for stroke (2009) Stroke thrombolysis in England, Wales and Northern Ireland: how much do we do and how much do we need? J Neurol Neurosurg Psychiatry 82:13-19. doi:10.1136/jnnp

3. The IST-3 collaborative group (2012) The benefits and harms of intravenous thrombolysis with recombinant tissue plasminogen activator within $6 \mathrm{~h}$ of acute ischaemic stroke (the third international stroke trial [IST-3]): a randomised controlled trial. Lancet 379(9834):2352-2363

4. Hacke W, Kaste M, Bluhmki E, Brozman M, Davalos A, Guidett D, Larrue V, Lees K, Medeghri Z, Machnig T, Schneider D, von Kummer R, Wahlgren N, and Toni D, for the ECASS Investigators (2008) Thrombolysis with Alteplase 3 to $4.5 \mathrm{~h}$ after Acute Ischemic Stroke. NEJM 359:1317-1329

5. Saver J (2007) Novel end point analytic techniques and interpreting shifts across the entire range of outcome scales in acute stroke trials. Stroke 38:3055-3062

6. IST 3 Collaborative Group (2012) Statistical analysis plan for the third International Stroke Trial (IST-3); part of a 'thread' of reports of the trial. Int J Stroke 7:186-187

7. Wardlaw J, Murray V, Berge E, del Zoppo G, Sandercock P, Lindley R, Cohen G (2012) r-tPA for acute ischaemic stroke: an updated systematic review and meta-analysis. Lancet 379: 2364-2372

8. Leys D, Cordonnier C (2012) rt-PA for ischaemic stroke: what will the next question be? Lancet 379:2320-2321 